\title{
Patient Reported Urinary Incontinence and Sexual Impotency Following Multimodality Radical Therapy for Prostate Cancer: An Untold Story of Compound Toxicity?
}

\section{Ori Haisraely}

Sheba Medical Center at Tel Hashomer: Sheba Medical Center

\section{Yaacov Richard Lawrence}

Sheba Medical Center at Tel Hashomer: Sheba Medical Center

Ron Lewin

Sheba Medical Center at Tel Hashomer: Sheba Medical Center

Orit Kaidar-Person

Sheba Medical Center at Tel Hashomer: Sheba Medical Center

llana Weiss

Sheba Medical Center at Tel Hashomer: Sheba Medical Center

Eyal Zimlichman

Sheba Medical Center at Tel Hashomer: Sheba Medical Center

\section{Raanan Berger}

Sheba Medical Center at Tel Hashomer: Sheba Medical Center

Zvi Symon ( $\nabla$ symonz@sheba.health.gov.il)

Chaim Sheba Medical Center: Sheba Medical Center at Tel Hashomer https://orcid.org/0000-0003-

0971-6921

\section{Research}

Keywords: patient reported outcomes, prostate cancer

Posted Date: December 29th, 2021

DOI: https://doi.org/10.21203/rs.3.rs-633279/v2

License: (c) (1) This work is licensed under a Creative Commons Attribution 4.0 International License.

Read Full License 


\section{Abstract}

Purpose: To evaluate urinary continence and sexual potency following radical prostatectomy and adjuvant radiotherapy.

Materials/Methods: Expanded Prostate Cancer Index Composite (EPIC) surveys of patients with localized prostate cancer treated with surgery followed by adjuvant/salvage pelvic radiotherapy (S+RT) were analyzed. A control cohort was primary radiotherapy (RT). Reverse "bifecta" was defined as a score less than 60 in both incontinence and sexual domains. Superior urinary function was defined as a score above 90 . The clinically important difference was calculated using a distribution approach.

Results: Surveys at least 1 year after treatment were available for $130 \mathrm{~S}+\mathrm{RT}$ and $374 \mathrm{RT}$ patients. For $\mathrm{S}+\mathrm{RT}$ vs. RT, the mean urinary incontinence score was 68 [6.25 -100] versus 86.4 [Cl-95 39.5-100] $(p<0.001)$, confirming 6.5 points of clinically significant difference. The adjusted odds ratio for superior urinary function was $2.67(1.7-4.1, p<0.001)$ for primary radiotherapy. The odds ratio of having both poor urinary and sexual performance (reverse " bifecta") was 0.29 in RT arm $(0.14-0.58, p<0.001)$ when adjusted for age and Androgen Deprivation Therapy (ADT) , group risk stratification, comorbidities and smoking status.

Conclusion: In this cross sectional study, Surgery with adjuvant/salvage RT was associated with significantly worse patient reported urinary continence outcomes at 1-year post treatment, lower odds of achieving perfect urinary continence and a threefold risk of reverse 'bifecta' with inferior urinary continence and sexual performance. Longitudinal studies of evolving toxicity are required to validate these findings. Patients referred for surgery with a high probability of requiring adjuvant or salvage radiotherapy should be informed regarding the potential composite toxicity of both surgery and radiotherapy.

\section{Introduction}

Cancer control, urinary continence and sexual function are the components of Trifecta, an outcome model for localized prostate cancer ${ }^{1}$. Since both overall survival and disease specific survival are comparable following modern surgery or radical radiotherapy ${ }^{2}$, disparate patient reported outcomes of urinary continence and sexual potency, hence "Bifecta", following primary therapy have been the subject of considerable controversy.

Patients failing primary therapy and requiring local salvage are often susceptible to compound toxicity in these domains. Long term study has shown that in tertiary center a $20-30 \%$ of patients treated with prostatectomy will require salvage radiotherapy ${ }^{3}$.Patients with high or very high risk prostate cancer who undergo radical prostatectomy are even more likely to require additional local therapy.Thus for example, using a widely utilized nomogram ${ }^{4}$, a 65 year old patient with T2B Gleason $4+4$ with $50 \%$ core positivity who undergoes radical prostatectomy is predicted to have a $20 \%$ chance of organ confined disease, and 
$79 \%$ chance of extracapsular extension. The nomogram offers an interpretation of this prediction reassuring that half of patients without organ confined disease will have long term control, however it is necessary to point out that the other half will require salvage radiotherapy. Thus, at least forty percent of men electing surgery with the above disease characteristics will be exposed to the compound toxicity of surgery and radiotherapy.

There is a growing body of literature regarding comparative domain specific outcomes of primary surgery versus primary radiotherapy ${ }^{2}$. These comparisons are confounded by multiple factors, including age, inherent differences in the toxicity profile, and differences in technique of both surgery and radiotherapy. For men who undergo surgery with a high probability of requiring radiotherapy, we propose that such comparisons are less meaningful. The composite effects of radical prostatectomy followed by salvage radiotherapy on quality of life are not generally discussed a-priori with the patient during the initial consultation even when the likelihood of requiring radiation is considerable.

In our study we endeavor to provide information regarding bifecta, urinary continence and sexual potency for patients with a high probability of requiring salvage radiation following surgery.

Quality of Life data routinely collected in an institutional review board (IRB) approved clinical database were utilized for this study. The specific goal of the current study was to evaluate one-year patientreported sexual and urinary outcomes of patients who underwent radical prostatectomy and salvage/adjuvant radiotherapy. For comparison, we studied a cohort of patients receiving primary radiation therapy.

\section{Material And Methods}

The cohort includes localized prostate cancer patients treated at the Sheba Medical Center, between the years 2002-2018 under the Sheba Medical Center Institutional Review Board approval for quality of life measurement and reporting of outcomes of genitourinary cancer (SMC-11-9132).

Patients were recruited by a research associate (IW) present at approximately one out of 4 outpatient genitourinary clinic days per week and asked to complete a paper survey. Patients who experienced difficulty were assisted. The survey included the validated Hebrew translation ${ }^{5}$ of the Expanded Prostate Cancer Index Composite short form (EPIC-26) measuring functional outcomes (urinary incontinence, urinary irritation and obstruction, bowel, sexual, and vitality and hormonal function) ${ }^{6}$, epidemiology data, and questions about the use of interventions for sexual dysfunction. For the purpose of the current study, we focused on urinary and sexual outcomes.

Multivariable regression models using the Statistical Package for the Social Sciences (SPSS) 24th edition, were utilized to compare functional outcomes diagnostic stages and self-reported treatment groups. 
In the surgery arm, patients underwent both robot-assisted laparoscopic prostatectomy or open radical prostatectomy. Since both kinds of surgery are associated with similar functional outcomes ${ }^{7}$, they were pooled for this analysis. Radiotherapy was performed in a single center and conformal 3-D radiotherapy was employed between 2002 and 2008, after which intensity modulated radiotherapy with daily image guidance was used. Adjuvant \Salvage radiotherapy was 70 Gy in 2 Gy or 64.4 Gy in 2/3 Gy fractions delivered to the prostate fossa with a $6 \mathrm{~mm}$ margin. Definitive radiotherapy was 78-82 $\mathrm{Gy}$ in $2 \mathrm{~Gy}$ fractions or $73.6 \mathrm{~Gy}$ in $2.3 \mathrm{~Gy}$ fractions delivered to the prostate and seminal vesicles with a $6 \mathrm{~mm}$ margin. High risk patients also received elective radiation to the pelvic nodes.

\section{Data Analysis}

Epic-26 a well-established validated tool for assessment of quality of life function in prostate cancer ${ }^{6}$.The four domains of assessment urinary, sexual, bowel, and hormonal domains were calculated as a score between 0-100 through a coding conversion to a SPSS analysis. Baseline patient's characteristics was collected by medical chart. Baseline characteristics were compared across treatments using T-test or Mann Whitney test for continuous parametric and a-parametric variables respectively. Categorical variables were analyzed using $\mathrm{X} 2$ test.

Each domain in the EPIC questionnaire was calculated for a score mean, median, range and standard deviation for each group of treatment. Scores distribution was analyzed for normalization criteria using histogram, kurtosis and skewness tests. None of the domains had normal distribution, so comparison was done by non-parametric analysis using Mann-Whitney test.

We also used distribution based approaches to establish minimally important (i.e., clinically-relevant) differences for each EPIC-26 domain. Previous studies have found that half of a standard deviation is appropriate choices for a distribution-based minimally important difference cut-point ${ }^{8}$.

A perfect urinary score (above 90 ) was then evaluated as a dichotomy variable. A Multivariable logistic regression was performed for treatment type, age, group risk, use of ADT, diabetes and smoking status. Adjusted Odds ratio (OR) and 95\% Confidence Interval (Cl) were calculated for each variable.

For the purpose of this quality of life study we have defined 'reverse bifecta' as sexual impotency and urinary incontinence with a score lower than 60 in both sexual and urinary domains of the EPIC ${ }^{9}$. This definition while new, correlates with previous studies that notice that a score below 60 is associated with worse QOL outcomes ${ }^{10}$. We calculated the odds of 'reverse bifecta' using multivariable logistic regression.

\section{Results}

\section{Patient characteristics}


The clinical database includes 2023 patients with prostate cancer treated with radiotherapy between 2002-2018, 1500 received radiotherapy (RT) and 523 surgery followed by adjuvant \salvage radiotherapy ( $\mathrm{S}+\mathrm{RT}$ ). A research associate (IW) attended one the outpatient clinics on one of four clinic days each week and approached 523 patients to complete surveys. Nineteen patients declined and 504 patients completed 1-year post treatment EPIC surveys, $130 \mathrm{~S}+\mathrm{RT}$ and 374 RT.

Patients epidemiologic and clinical data are reported in Table 1.

Table 1

-Patient and disease characteristics.

\begin{tabular}{|llll|}
\hline & RT & S+RT & P value \\
\hline $\mathrm{n}$ & $74 \%(374)$ & $26 \%(130)$ & \\
\hline Age & $69.8 \pm 6.6$ & $64 \pm 6.6$ & $<0.001^{*}$ \\
\hline Smoking status & $6.9 \%$ & $4.7 \%$ & 0.37 \\
\hline Married/in relationship & $90.3 \%$ & $96.1 \%$ & $0.041^{*}$ \\
\hline Diabetes mellitus & $25.5 \%$ & $18.6 \%$ & 0.1 \\
\hline Ischemic heart disease & $19.7 \%$ & $11.7 \%$ & $0.042^{*}$ \\
\hline Cerebrovascular disease & $5.5 \%$ & $2.3 \%$ & 0.146 \\
\hline Peripheral vascular disease & $8.8 \%$ & $7 \%$ & 0.52 \\
\hline Chronic renal failure & $8.5 \%$ & $3.1 \%$ & $0.042^{*}$ \\
\hline Respiratory illness & $12.1 \%$ & $10 \%$ & 0.54 \\
\hline Gastrointestinal conditions & $12.6 \%$ & $14 \%$ & 0.69 \\
\hline Depression & $6.8 \%$ & $4.7 \%$ & 0.38 \\
\hline Drug abuse & $0.3 \%$ & $0.8 \%$ & 0.4 \\
\hline PSA levels before radiation & $16.8 \pm 30.5$ & $1.5 \pm 7.1$ & $<0.001^{\star}$ \\
\hline Risk stratification & $184(49.5 \%)$ & $78(60 \%)$ & $0.021^{*}$ \\
\hline High & $147(29.3 \%)$ & $47(36.2 \%)$ & \\
\hline Intermediate & $41(11 \%)$ & $5(3.8 \%)$ & \\
\hline low & $58.7 \%$ & & \\
\hline ADT use & & $37.5 \%$ & \\
\hline $\begin{array}{l}\text { Patient's characteristics. Comparison between radiotherapy and surgery + radiotherapy. Statistical } \\
\text { analysis using t-test and Anova for parametric variable and Mann-Whitney and Kruskal-Wallis for } \\
\text { non-parametric variables. * statistical significant }\end{array}$ & & \\
\hline
\end{tabular}


The mean age for RT was older compare with S+RT with a mean age was $69.8 \pm 6.6$ vs. $64 \pm 6.6(P<0.001)$ respectively. There was no difference in smoking status diabetes, peripheral vascular, respiratory, or gastrointestinal diseases. There was a statistical significant difference for ischemic heart disease $(19.7 \%$ vs $11.8 \%$. $p=0.042)$ and chronic renal disease $(8.5 \%$ vs $3.1 \%, p=0.042)$.

The National Comprehensive Cancer consortium group risk classification between both cohorts was statistically different. High, intermediate and low risk were $49.5 \%, 29.3 \%$ and $11 \%$ in the RT group respectively and $60 \%, 36 \%$ and $4 \%$ in the $S+R T$ group respectively. $(P=0.021)$. There was more Androgen deprivation therapy (ADT) use in the RT group $(58.7 \%)$ as compare to the RP+RT cohort $(p<0.001)$.

\section{EPIC domain scores}

The mean urinary incontinence score was $68 \pm 32$ [6.25 -100] for S+RT versus $86.4 \pm 19$ [Cl-95 39.5-100] for RT $(p<0.001)$. (Table 2). Using the distribution approach with a 0.5 SD this result confirms a 6.5 points of clinically significant difference. The urinary irritation domain was not statistical different between the groups, but the overall urinary function was higher in the RT group ( 82.6 vs $74.8, p=0.002$ ). 
Table 2

-EPIC domain scores

\begin{tabular}{|c|c|c|c|}
\hline & RT & S+RT & $\mathrm{p}$ value \\
\hline$n$ & 374 & 130 & \\
\hline \multicolumn{4}{|l|}{ SCORE Mean \pm SD } \\
\hline \multicolumn{4}{|l|}{ Median (range) } \\
\hline \multirow[t]{2}{*}{ Urinary } & $82.6 \pm 21.2$ & $74.8 \pm 23.6$ & $0.002^{\star}$ \\
\hline & $88(11.1-100)$ & $81.5(9.2-100)$ & \\
\hline \multirow[t]{2}{*}{ Urinary incontinence } & $86.4 \pm 19$ & $68 \pm 32$ & $0.001^{*}$ \\
\hline & $100(0-100)$ & $75(0-100)$ & \\
\hline \multirow[t]{2}{*}{ Urinary irritation } & $81.5 \pm 19$ & $83.5 \pm 20$ & 0.134 \\
\hline & $87.5(6.25-100)$ & $87.5(12.5-100)$ & \\
\hline \multirow[t]{2}{*}{ Sexual } & $28.5 \pm 25$ & $31.3 \pm 29.1$ & 0.576 \\
\hline & $16.6(0-100)$ & $18(0-100)$ & \\
\hline \multirow[t]{2}{*}{ Hormonal } & $83.8 \pm 20.6$ & $89.5 \pm 16$ & $<0.001^{\star}$ \\
\hline & $90(0-100)$ & $100(5-100)$ & \\
\hline \multirow[t]{2}{*}{ Bowel } & $87.1 \pm 18.1$ & $91.1 \pm 16$ & $P=0.009 *$ \\
\hline & $95.8(0-100)$ & $100(8.3-100)$ & \\
\hline
\end{tabular}

There was no statistical difference in sexual domains between the two cohorts. For the S+RT the mean score was $31.3 \pm 29.1$ and for the RT group the mean score was $28.5 \pm 25(p=0.576)$ (Table 3 ) 
Table 3

Multivariate regression for superior urinary function.

\begin{tabular}{|lll|}
\hline Variable & OR (Cl 95\%) & p value \\
\hline Age $>69$ & $0.95(0.72-1.53)$ & 0.75 \\
\hline Androgen deprivation therapy & $0.97(0.6-1.52)$ & 0.132 \\
\hline High risk group & $0.88(0.6-1.29)$ & 0.78 \\
\hline Diabetes & $0.6(0.4-0.91)$ & $0.025^{\star}$ \\
\hline Current smoker & $0.84(0.4-1.76)$ & 0.71 \\
\hline Definitive radiotherapy & $2.67(1.7-4.1)$ & $<0.001^{*}$ \\
\hline
\end{tabular}

The hormonal score was significantly worse in the primary radiotherapy cohort with a mean score of $83.3 \pm 20.6$ compared with $89.5 \pm 16$ in the surgery arm $(p<0.001)$. Moreover, the bowel function score was also significantly lower in the primary radiotherapy relatively to surgery arm with a score of $87.1 \pm 18.1$ and $91.1 \pm 16$ respectively $(\mathrm{p}=0.009)$.

\section{Multivariable analysis}

Multivariable regression analysis was performed for superior urinary score Adjustment for age (as a dichotomy variable), risk group (high risk group for reference), ADT use and treatment type (RT for reference) were performed. The adjusted Odds ratio for superior urinary function was 2.67 (1.7-4.1, $p<0.001$ ) for definitive radiotherapy. (Table 4.)

Table 4

Multivariate regression for "reverse bifecta" defined as a score less than 60 in both incontinence and sexual domains of the EPIC.

\begin{tabular}{|lll|}
\hline Categories & OR (Cl 95\%) & P value \\
\hline Age $>69$ & $1.8(0.9-3.7)$ & 0.125 \\
\hline Androgen deprivation therapy & $2(0.95-5)$ & 0.081 \\
\hline High risk group & $1.53(0.76-3.3)$ & 0.47 \\
\hline Diabetes & $1.69(0.78-3.3)$ & 0.23 \\
\hline Current smoker & $1.38(0.38-4.9)$ & 0.69 \\
\hline Definitive radiotherapy & $0.29(0.14-0.58)$ & $<0.001$ * \\
\hline
\end{tabular}

Multivariable regression analysis was also performed for the 'reverse bifecta' as a dichotomy variable. The odds ratio of having both poor urinary and sexual performance was $0.29(0.14-0.58, \mathrm{P}<0.001)$ for primary radiotherapy when adjust to the same variables. (Table 4).

\section{Discussion}


In this multivariable analysis, one-year urinary domain quality of life scores after prostatectomy with salvage/adjuvant radiotherapy were significantly worse compared to patients undergoing primary radiotherapy. An estimate of the clinical significance of this discrepancy using the standard deviation and distribution approach revealed a 6.5-point difference in the urinary domain which has been previously shown to be a clinically important difference ${ }^{8}$.

While the overall urinary function was indeed worse in the surgery, when dividing this domain into incontinence and irritate/obstruction domains the results were different. In the incontinence domain as well documented, primary radiotherapy was significantly better, as radiation is more forgiving regarding sphincter function than surgical reconstruction. In the obstruction and irritation domain, there was no difference between the cohorts as radiation was delivered in both cohorts. While surgery alone is associated with less urinary irritation than radiotherapy, patients who undergo both modalities will more likely suffer from both incontinence and irritation ${ }^{11-13}$.

Despite the fact that the cohort of primary radiotherapy comprised older subjects and the use of ADT was higher as compare to the surgery arm, there was no significantly difference in sexual performance. Furthermore, most importantly for patient education prior to choice of treatment for high risk prostate cancer, the odds of achieving both worse sexual and urinary function were threefold for the multimodality radical surgery and radiation cohort versus radiotherapy alone. This finding was sustained after adjusting for age, diabetes, and risk group. This is despite a significantly worse hormonal score in the primary radiotherapy cohort due to a higher rate of ADT. Bowel function score was marginally worse in the primary radiotherapy arm, possibly due to a higher radiation dose to the rectum in primary radiotherapy arm compared to salvage radiotherapy. This difference was lower than the distribution-based minimally important difference.

Few studies comprehensively address urinary function following post prostatectomy radiotherapy. A literature search, reveals retrospective studies that compared surgery alone to post prostatectomy radiation. A quality of life decrement of $10 \%$ was reported when radiation was added to prostatectomy, which is further reduced by androgen deprivation therapy ${ }^{14}$. The authors suggest that patients with high risk disease "should be counseled before RP on the potential net impairment of functional outcomes arising from multimodal treatment." An odds ratio of 1.6 for urinary incontinence has been reported for the combination compared to surgery alone ${ }^{15}$. A limitation of most studies is that urinary incontinence was reported by the number of pads used in 24 hours rather than a comprehensive patient reported domain for urinary incontinence ${ }^{14-16}$. In particular, no other study compared quality of life outcomes of surgery with adjuvant/salvage radiation versus primary radiation therapy with or without androgen deprivation therapy.

There are a number of limitations to the current study. Firstly, pre-treatment baseline QOL measurement was not collected in the surgical cohort, thus precluding a report of longitudinal quality of life outcomes or a comparison with a surgery alone cohort. This limitation is however addressed well by comparing the mean scores for EPIC in the study by Sanda et al ${ }^{17}$, which demonstrated the same mean urinary 
incontinence and sexual domains scores at one year follow up for primary radiotherapy. The historical mean urinary incontinence score of the surgery alone cohort was 78 in the latter study, versus 68 for the combined surgery and radiotherapy cohort and 86.4 for the radiation only cohort in this study, thus supporting detection of a clinically significant difference. Secondly, it may be argued that a one-year time point following radiotherapy does not capture all late effects. However, one year status of urinary outcomes is fairly predictive of later outcomes and very late onset of incontinence in patients with good outcomes at one year is uncommon ${ }^{18}$. Thirdly, only one quarter of patients in the database were requested to complete surveys selected by the presence of the research associate on one of four clinic days. Thus while it may be argued that a capture rate of approximately one quarter of the patients may introduce a bias, this was not due to a patient selection bias as $95 \%$ of patients asked to fill surveys complied. Furthermore, the capture rate was similar in both the study group and the control arm, thus confirming an absence of selection bias and the risk of a skewed view of outcomes.

This study provides important information for both patients and physicians asked to guide choice of treatment since it has a number of important strengths; Firstly, these are patient reported outcomes and not physician reported toxicity, in a sizeable cohort of real life subjects without exclusion criteria. Second, the statistical methods used in this paper were multivariable logistic regression with robust consideration of multiple confounders. We utilized clinical significant difference metrics, and while this approach is more common in a longitudinal study, it supports a significant difference in population based mean scores. Finally, it is becoming increasingly important to report patient reported outcomes specific to an institution as radiotherapy volumes and techniques vary considerably, particularly in the post prostatectomy setting. Patient reported outcomes from a specific institution can provide a realistic expectation based on the local clinical expertise and thus is more pertinent than outcomes reported from other centers ${ }^{19}$.

\section{Conclusions}

In conclusion, surgery with adjuvant/salvage RT was associated with significantly worse patient reported urinary continence outcomes at one-year post treatment, lower odds of achieving perfect urinary continence and higher odds of both unfavorable urinary and sexual performance. Patients referred for surgery with a high probability of requiring adjuvant or salvage radiotherapy should be informed regarding the potential impact on urinary and sexual performance due to the composite effect of two radical treatments, surgery and radiotherapy. These patients should always be referred to an oncologist to discuss the option of primary radiotherapy with or without ADT, which was associated with more favorable 'bifecta' in this study. These findings require further investigation and confirmation with longer follow-up and longitudinal quality of life outcomes.

\section{Abbreviations}

EPIC- Expanded Prostate Cancer Index $\underline{\text { Composite }}$ 
S+RT- Surgery and Radiotherapy (surgery followed by adjuvant/salvage pelvic radiotherapy)

RT- Radiotherapy (Primary radiotherapy)

ADT - Androgen deprivation therapy

IRB -institutional review board

SMC- Sheba Medical Center

IW- Ilana Weiss

3-D Three dimensional

SPSS- Statistical Package for the Social Sciences

QOL Quality of Life

Gy-Gray

mm- milimeter

OR- Odds ratio

Cl- Confidence Interval

\section{Declarations}

Ethics approval and consent to participate: Study was performed with ethics approval from the Sheba Medical Center Institutional Review Board approval for quality of life measurement and reporting of outcomes of genitourinary cancer (SMC-11-9132).

Consent for publication: The ethics board at Sheba SMC-11-9132 does not require specific patient consent as the quality of life data are used clinically to improve patient care and then anonymized prior to analysis and thus publications from this database are exempt

Availability of data and materials- The datasets used and/or analysed during the current study are available from the corresponding author on reasonable request.

Competing interests- The authors declare they have no competing interests.

Funding- This study was partially funded by a Parasol Foundation grant

Author contributions:

Ori Haisraely - concept, data analysis, statistics, manuscript preparation 
Yaacov Richard Lawrence - review statistics and manuscript

Ron Lewin - data collection, manuscript review

Orit Kaidar-Person- manuscript review, scientific editing.

Ilana Weiss- data collection, database manager

Eyal Zimlichman- manuscript review

Raanan Berger- manuscript review

Zvi Symon - concept, data analysis, statistics, manuscript preparation

Acknowledgements: Tamara Kushnir MA and Iris Oren Ivri BA, nurse specialists for their clinical contribution.

\section{References}

1. Jereczek-Fossa BA, Zerini D, Fodor C, et al. Reporting combined outcomes with Trifecta and survival, continence, and potency (SCP) classification in 337 patients with prostate cancer treated with imageguided hypofractionated radiotherapy. BJU Int. Dec 2014;114(6b):E3-E10. doi:10.1111/bju.12530

2. Hamdy FC, Donovan JL, Lane JA, et al. 10-Year Outcomes after Monitoring, Surgery, or Radiotherapy for Localized Prostate Cancer. N Engl J Med. Oct 13 2016;375(15):1415-1424. doi:10.1056/NEJMoa1606220

3. van Dessel LF, Reuvers SHM, Bangma CH, Aluwini S. Salvage radiotherapy after radical prostatectomy: Long-term results of urinary incontinence, toxicity and treatment outcomes. Clin Transl Radiat Oncol. Jun 2018;11:26-32. doi:10.1016/j.ctro.2018.05.001

4. Katz MS, Efstathiou JA, D'Amico AV, et al. The 'CaP Calculator': an online decision support tool for clinically localized prostate cancer. BJU Int. May 2010;105(10):1417-22. doi:10.1111/j.1464410X.2010.09290.x

5. Kushnir T, Gofrit ON, Elkayam R, et al. Impact of Androgen Deprivation Therapy on Sexual and Hormonal Function in Patients Receiving Radiation Therapy for Prostate Cancer. Isr Med Assoc J. Jan 2016;18(1):49-53.

6. Wei JT, Dunn RL, Litwin MS, Sandler HM, Sanda MG. Development and validation of the expanded prostate cancer index composite (EPIC) for comprehensive assessment of health-related quality of life in men with prostate cancer. Urology. Dec 20 2000;56(6):899-905. doi:10.1016/s00904295(00)00858-x

7. Coughlin GD, Yaxley JW, Chambers SK, et al. Robot-assisted laparoscopic prostatectomy versus open radical retropubic prostatectomy: 24-month outcomes from a randomised controlled study. Lancet Oncol. Aug 2018;19(8):1051-1060. doi:10.1016/S1470-2045(18)30357-7 
8. Skolarus TA, Dunn RL, Sanda MG, et al. Minimally important difference for the Expanded Prostate Cancer Index Composite Short Form. Urology. Jan 2015;85(1):101-5.

doi:10.1016/j.urology.2014.08.044

9. Spiess PE, Given RW, Jones JS. Achieving the 'bifecta' using salvage cryotherapy for locally recurrent prostate cancer: analysis of the Cryo On-Line Data (COLD) registry data. $B J U$ Int. Jul 2012;110(2):217-20. doi:10.1111/j.1464-410X.2011.10659.x

10. Levinson AW, Ward NT, Pavlovich CP. Defining potency: a comparison of the International Index of Erectile Function short version and the Expanded Prostate Cancer Index Composite. Cancer. Dec 1 2009;115(23):5608. doi:10.1002/cncr.24613

11. Lee TK, Breau RH, Mallick R, Eapen L. A systematic review of expanded prostate cancer index composite (EPIC) quality of life after surgery or radiation treatment. Can J Urol. Feb 2015;22(1):7599606.

12. Chen RC, Basak R, Meyer AM, et al. Association Between Choice of Radical Prostatectomy, External Beam Radiotherapy, Brachytherapy, or Active Surveillance and Patient-Reported Quality of Life Among Men With Localized Prostate Cancer. JAMA. Mar 21 2017;317(11):1141-1150. doi:10.1001/jama.2017.1652

13. Barocas DA, Alvarez J, Resnick MJ, et al. Association Between Radiation Therapy, Surgery, or Observation for Localized Prostate Cancer and Patient-Reported Outcomes After 3 Years. JAMA. Mar 21 2017;317(11):1126-1140. doi:10.1001/jama.2017.1704

14. Adam M, Tennstedt P, Lanwehr D, et al. Functional Outcomes and Quality of Life After Radical Prostatectomy Only Versus a Combination of Prostatectomy with Radiation and Hormonal Therapy. Eur Urol. Mar 2017;71(3):330-336. doi:10.1016/j.eururo.2016.11.015

15. Suardi N, Gallina A, Lista G, et al. Impact of adjuvant radiation therapy on urinary continence recovery after radical prostatectomy. Eur Urol. Mar 2014;65(3):546-51. doi:10.1016/j.eururo.2013.01.027

16. Ervandian M, Hoyer M, Petersen SE, et al. Late urinary morbidity and quality of life after radical prostatectomy and salvage radiotherapy for prostate cancer(). Scand J Urol. Dec 2017;51(6):457463. doi:10.1080/21681805.2017.1354314

17. Sanda MG, Dunn RL, Michalski J, et al. Quality of life and satisfaction with outcome among prostatecancer survivors. N Engl J Med. Mar 20 2008;358(12):1250-61. doi:10.1056/NEJMoa074311

18. Whi JT et al, Comprehensive Comparison of Health-Related Quality of Life After Contemporary Therapies for Localized Prostate Cancer, J Clin Oncol 2002, 20:557-566

19. Mathieu R, Arango JD, Beckendorf V, et al. Nomograms to predict late urinary toxicity after prostate cancer radiotherapy. World J Urol. Jun 2014;32(3):743-51. doi:10.1007/s00345-013-1146-8

20. Kluetz PG, Chingos DT, Basch EM, Mitchell SA. Patient-Reported Outcomes in Cancer Clinical Trials: Measuring Symptomatic Adverse Events With the National Cancer Institute's Patient-Reported Outcomes Version of the Common Terminology Criteria for Adverse Events (PRO-CTCAE). Am SoC Clin Oncol Educ Book. 2016;35:67-73. doi:10.1200/edbk_159514 